\section{Catherine Lavau Joop Jansen Karsten Weis Angus Lamond Anne Dejean}

\section{ADRESSES}

C. Lavau : étudiante en thèse. J. Jansen : stagiaire post-doctoral. A. Dejean : directeur de recherche à l'Inserm. Unité de recombinaison et expression génétique, Inserm U.163, Institut Pasteur, 28, rue du Docteur-Roux, 75724 Paris Cedex 15, France. K. Weis étudiant en thèse. A. Lamond : chef de laboratoire. EMBL, Meyerhof strasse 1, D69117 Heidelberg, Allemagne.

\title{
Leucémie aiguë promyélocytaire et acide rétinoïque : le paradoxe
}

La leucémie aiguë promyélocytaire est associée à une translocation chromosomique $\mathbf{t}(15 ; 17)$, qui juxtapose les gènes PML (promyelocytic leukemia) et $R A R \alpha$ (récepteur de l'acide rétinoïque $\alpha$ ) et entraîne la synthèse d'une protéine hybride PML-RAR. La présence de cet hybride empêche l'action des rétinoïdes sur la différenciation de la lignée promyélocytaire qui reste bloquée à un stade précoce. L'hybride interfère avec plusieurs voies d'activation de la transcription, liées non seulement à RAR, mais aussi à RXR, et auX récepteurs des hormones thyroïdiennes et de la vitamine $D$. Paradoxalement, l'acide rétinoïque lève ce blocage et induit la différenciation, aboutissant à la rémission morphologique complète de la leucémie, avec, en particulier, réorganisation des structures nucléaires et réapparition des corps nucléaires.

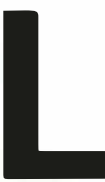

a leucémie aiguë promyélocytaire (LAP) a suscité un intérêt croissant depuis les cinq dernières années, qui dépasse largement le seul champ de l'hématologie. En effet, deux propriétés, spécifiques de ce type d'hémopathie, ont attiré l'attention des spécialistes de différents secteurs de recherche : tout d'abord, la rémission de la maladie obtenue par le traitement à l'acide rétinoïque $(\mathrm{AR})$, car la grande sensibilité des cellules blastiques promyélocytaires aux rétinoïdes fait de cette leucémie un modèle unique de thérapie par différenciation [1] ; par ailleurs, la présence d'une translocation chromosomique qui est devenue un marqueur diagnostique de la maladie [2]. Cette translocation juxtapose un gène jusqu'alors inconnu, PML (promyelocytic leukemia), au gène codant pour le récepteur $\alpha$ de l'acide rétinoïque $(\operatorname{RAR} \alpha)$ (pour revue, voir [3-5]). La protéine hybride PML-RAR $\alpha$ résultant de cette fusion est actuellement la cible de nombreuses recherches. L'implication d'un mutant naturel d'un récepteur de l'acide rétinoïque dans les LAP apparaît tout à fait paradoxal. D'une part, l'altération de la voie d'activation par les réti- 


\section{RÉFÉRENCES}

1. Degos L, Castaigne $S$, Fenaux $P$ Daniel MT, Chomienne C. Le traitement des leucémies aiguës à promyélocytes par l'acide tout-trans rétinoïque. médecine/ sciences $1991 ; 7$ : 460-4.

2. Larson RA, Kondo K, Vardiman JW, Butler AE, Golomb HM, Rowley JD. Evidence for a 15;17 translocation in every patient with acute promvelocytic leukemia. Am J Med $1984 ; 76: 827-41$.

3. Grignani F, Fagioli $\mathbf{M}$, Alcalay $\mathbf{M}$, Longo L, Pandolfi PP, Donti E, Biondi A, Lo Coco F, Grignani F, Pelicci PG. Acute promyelocytic leukemia: from genetics to treatment. Blood $1994 ; 83: 10-25$.

4. Warrell RP, de Thé $H$, Wang $Z Y$, Degos L. Acute promyelocytic leukemia. $N$ Engl J Med 1993 ; 329 : 177-89.

5. de Thé H, Koken M, Stadler M, Daniel MT, Puvion E, Chomienne C, Degos L. Un nouveau compartiment nucléaire, révélé par des autoanticorps de la cirrhose biliaire primitive, pourrait être impliqué dans la pathogénie de la leucémie aiguë
promyélocytaire. méderine/sciences 1994 ; promyélocy
$10: 577-82$.

6. Tabin CJ. Retinoids, homeoboxes and growth factors : toward molecular models growth factors : toward molecular models
for limb development. Cell 1991 ; 66 : 199 .

7. Leid M, Kastner P, Chambon P. Multiplicity generated diversity in the retinoic acid signalling pathways. Trends Biol Sci 1992 ; $17: 427-33$.

8. Pratt MAC, Kralova J, McBurney MW. A dominant negative mutation of the $\alpha$ retinoic acid receptor gene in a retinoic acid-nonresponsive embryonal carcinoma cell. Mol Cell Biol 1990; 10 : 6445.

9. Robertson KA, Emani B, Collins SJ Retinoic acid-resistant HL-60R cells harbor a point mutation in the retinoic acid receptor ligand binding domain that confers dominant negative activity. Blood $1989 ; 80: 1885$.

10. Kruyt FAE, Van der Verr LJ, Mader $S$, Van den Brink CE, Feijen A, Jonk LJC, Kruijer W, Van der Saag PT. Retinoic acid resistance of the variant embryonal carcinoma cell line RAC65 is caused by expression of a truncated RAR $\alpha$. Differentiation $1992 ; 49: 27$

11. Collins SJ, Robertson K, Mueller L. Retinoic acid induced granulocytic differentiation of HL60 mveloid leukemi cells is mediated directly through the retinoic acid receptor $(\operatorname{RAR} \alpha)$. Mol Cell Biol $1990 ; 10: 2154$.

12. Tsai S, Collins SJ. A dominant negative retinoic acid receptor blocks neutrophil differentiation at the promvelocyte stage. Proc Nall Acad Sci USA 1993 ; 90 : 7153-7.

13. Evans RM. The steroid and thyroid hormone receptor superfamily. Science 1988 ; $240: 889-95$.

14. Beato M. Gene regulation by steroid noïdes due à la présence de l'hybride PML-RAR $\alpha$ contribue très probablement au phénotype leucémique et, en particulier, au blocage des promyélocytes à un stade précoce de leur différenciation. D'autre part, l'activation de cette même voie par l'AR entraîne une induction de la différenciation vers la voie granulocytaire. Ainsi, il est probable que la chimère-PML-RAR $\alpha$ puisse être responsable à la fois du blocage de la différenciation et de la sensibilité particulière à l'acide rétinoïque. La leucémie aiguë promyélocytaire serait de ce fait la première néoplasie à pouvoir ainsi bénéficier d'une thérapie directement ciblée vers la protéine oncogénique.

\section{Structure et fonction du récepteur $\alpha$ de l'acide rétinoïque}

La vitamine A et ses dérivés, principalement les acides rétinoïques touttrans et 9-cis, exercent des effets pléiotropiques sur le développement et la différenciation cellulaire [6]. La plupart des effets biologiques des rétinoïdes sont relayés par des récepteurs nucléaires. Deux familles de récepteurs de l'acide rétinoïque ont été décrites : les RAR proprement dits, dont les ligands sont indifféremment les AR tout-trans ou 9-cis, et les RXR qui ne lient que l'AR 9-cis (pour revue, voir [7]). Chaque famille comprend trois membres pour lesquels codent des gènes différents, $\alpha$, $\beta$, et $\gamma$ très bien conservés au cours de l'évolution. La multiplicité de ces récepteurs explique probablement la diversité des effets biologiques des rétinoïdes ; il est cependant encore difficile d'attribuer une fonction précise à l'un d'entre eux.

Un certain nombre de travaux suggèrent que $\mathrm{RAR} \alpha$ pourrait être impliqué dans la différenciation cellulaire. Les lignées en culture, telles que la lignée de tératocarcinome murin F9 ou myéloïde humaine HL60, ont la capacité de se différencier en présence d'AR. Des mutants devenus résistants au traitement ont été isolés pour chaque lignée et il s'est avéré que ces mutants contiennent toujours un RAR $\alpha$ altéré $[8,9]$. Par ailleurs, l'introduction d'un RAR $\alpha$ natif par transfection ou par infection rétrovirale dans chacune de ces lignées est capable de restaurer la sensibilité à l'AR $[10,11]$. Enfin, l'expression d'un mutant RAR $\alpha$ dominant négatif - c'est-à-dire capable d'inhiber la fonction du récepteur normal -, dans des cellules de moelle murines bloque la différenciation granulocytaire au stade promyélocytaire [12].

Les récepteurs de l'acide rétinoïque appartiennent à la grande famille des récepteurs nucléaires, composée d'un ensemble de facteurs de transcription dont la fonction est inductible par un ligand (hormones stéroïdes, vitamine $\mathrm{D}_{3}$, hormone thyroïdiemne...) ( $m / s n^{\circ} 2$, vol. 5, p. 125 $[13,14])$. Leur organisation modulaire comporte, très schématiquement, cinq régions principales correspondant à des domaines fonctionnels distincts. Tout d'abord, la région amino-terminale $\mathrm{A} / \mathrm{B}$ qui, sans correspondre à un réel domaine transactivateur, est capable de moduler la transcription, et cela selon le type cellulaire et le promoteur étudiés. La région $C_{\text {, }}$, riche en résidus cystéine, qui correspond au domaine de liaison à l'ADN, est la mieux conservée entre tous les récepteurs nucléaires (de l'ordre de $50 \%$ d'identité). Elle renferme deux "doigts de zinc", motifs protéiques dans lesquels un atome de zinc échangeant des liaisons de coordination avec 2 His et 2 Cys ou avec 4 Cys, stabilise une conformation en doigt [15] et correspond au domaine de liaison à l'ADN. Des résultats très récents ont, par ailleurs, permis de démontrer que cette région est le siège d'interactions protéiques déterminant la spécificité du choix de la cible [16-18]. La région E est complexe car elle renferme non seulement le domaine de liaison du ligand et le domaine transactivateur majeur - dont la fonction est inductible par le ligand -, mais elle contient aussi une interface de dimérisation qui confère au récepteur l'aptitude à former des dimères en solution. La fonction des régions $\mathrm{D}$ et $\mathrm{F}$, qui sont très peu conservées entre les différents récepteurs, est encore obscure. La seule information concerne la présence d'un certain nombre d'acides aminés de type basique au sein de la région $\mathrm{D}$, correspondant très probablement à un signal de localisation nucléaire. 


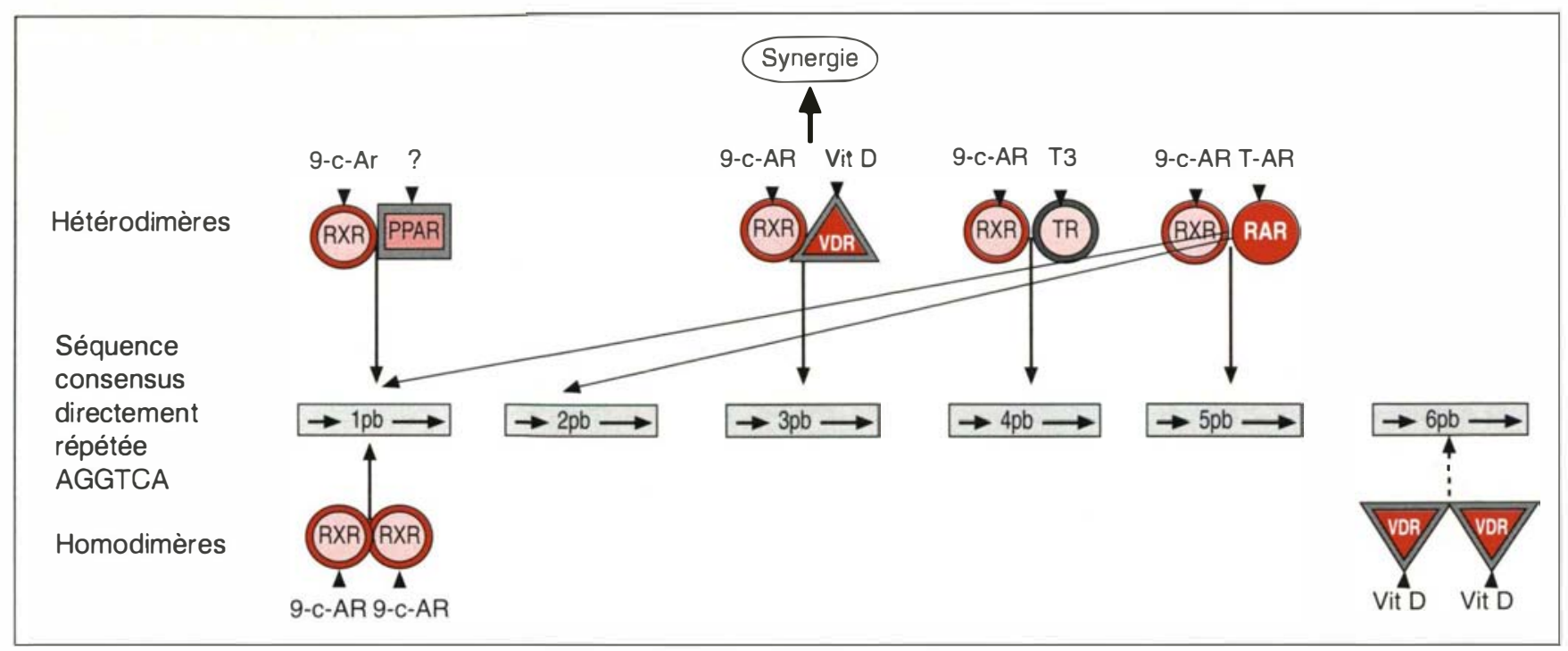

Figure 1. Spécificité de liaison des récepteurs nucléaires à leurs cibles. Les récepteurs nucléaires, sous la forme $d^{\prime}$ un hétérodimère avec la molécule $R X R$, lient un motif $d^{\prime} A D N$, présent dans la région promoteur des gènes cibles, composé d'une séquence consensus (AGGTCA) directement répétée et espacée par un nombre variable de nucléotides (de 1 à $6 \mathrm{pb}$ ). L'espacement entre les deux motifs répétés spécifie le type de réponse hormonale. RXR : récepteur de l'acide rétinoïque 9-cis (9-c-AR), PPAR : récepteurs activés par les proliférateurs de peroxysome et les acides gras, VDR : récepteur de la vitamine $D_{3}$, TR : récepteur de l'hormone thyroïdienne $T_{3}$, RAR : récepteur de I'acide rétinoïque tout-trans (T-AR).

Un certain nombre d'éléments de réponse à l'acide rétinoïque (RARE) ont été identifiés dans la région promoteur des gènes cibles de l'AR $\left(m / s n^{\circ} 3\right.$, vol. 6, p. 307). Cependant, la liaison des seuls RAR au niveau de ces séquences est peu efficace et le véritable complexe liant l'ADN avec une forte affinité est un hétérodimère RAR/RXR (m/s $n^{\circ} 8$, vol. 8, p. 879) (figure 1). La protéine RXR, qui est aussi capable de former des hétérodimères avec les récepteurs de l'hormone thyroïdienne (TR), de la vitamine $\mathrm{D}_{3}$ (VDR) ainsi qu'avec les récepteurs activés par les proliférateurs de peroxysome (PPAR), jouerait ainsi le rôle majeur de partenaire " universel" au sein de voies d'activation hormonale multiples [19]. En effet, il existe maintenant un certain nombre d'arguments en faveur de la réalité biologique de tels hétérodimères in vivo. Cette hétérodimérisation avec un partenaire RXR est, par ailleurs, conservée au cours de l'évolution car on retrouve un homologue de RXR chez la drosophile. Il s'agit de la protéine ultraspiracle (USP), qui forme effectivement un hétérodimère actif avec le seul récepteur nucléaire commu actuellement chez l'insecte, le récepteur de l'ecdrsone [20-22]. Une séquence consensus pour les différents éléments de réponse hormonaux a été établie : il s'agit d'un motif de type AGGTCA, directement répété (DR), avec un espacement variable entre les deux demi-palindromes allant de 1 à 5 nucléotides. Il a été proposé que l'espacement entre les deux motifs répétés correspondrait à une sorte de code discriminatif pour la reconnaissance du choix de la cible (figure 1). Ainsi un espacement de 5 (DR5), 4 (DR4), 3 (DR3) et l nucléotide(s) (DRl) correspondrait respectivement à un élément de réponse à l'AR, à l'hormone thyroïdiemne, à la vitamine $\mathrm{D}_{3}$ et aux proliférateurs de peroxysome [23]. Cependant, ce code de recomnaissance est relativement dégénéré en ce qui concerne les RAR car un certain nombre d'éléments de réponse de type DRl et DR2 ont aussi été identifiés dans le promoteur de gènes contrôlés par l’AR [24]. Par ailleurs, les choses se compliquent un peu plus lorsque l'on sait que le ligand du récepteur RXR, l'acide rétinoïque 9-cis, est capable d'induire la formation d'homodimères RXR-RXR qui reconnaissent plus particulièrement les motifs DRl [25]. De même, des travaux récents laissent supposer l'existence d'homodimères du récepteur de la vitamine $D_{3}$ qui fixeraient spécifiquement la séquence directement répétée espacée de 6 nucléotides [26]

\section{La protéine PML}

La fonction de la protéine PML native est encore inconnue et, jusqu'à présent, les seules informations concernent son analogie avec un certain nombre d'autres protéines ainsi que son très particulier profil d'expression intracellulaire. Elle appartient à une famille de protéines nouvellement décrites, caractérisées par la présence d'un motif riche en résidus cystéine/histidine de type doigt de zinc, appelé $R I N G$ finger, qui pourrait correspondre à un domaine de liaison aux acides 


\section{RÉFÉRENCES}

15. Klug A, Rhodes D. "Zinc fingers": a novel protein motif for nucleic acid recognition. Trends Biol Sci $1987 ; 12: 464$ 9.

16. Perlmann $T$, Rangarajan PN, Umesono K, Evans RM. Determinants for selective RAR and TR recognition of direct repeat HREs. Genes Dev 1993 ; 7 : 1411-22.

17. Kurokawa R, Yu VC, Näär A, Kyakumoto $S$, Han $Z$, Silverman $S$ Rosenfeld MG, Glass CK. Differential orientations of the DNA-binding domain and carboxyterminal dimerization interface regulate binding site selection by nuclear receptor heterodimers. Genes Dev 1993 ; 7 : 1423-35.

18. Mader S, Chen JY, Chen Z, White J, Chambon $P$, Gronemeyer $H$. The patterns of binding of RAR, RXR and TR homo and heterodimers to direct repeats are dictated by the binding specificities of the DNA binding domains. EMBO J $1993 ; 12$ : 502941.

19. Green S. Promiscuous liaisons. Nature $1993 ; 361: 590-1$.

20. Thomas HE, Stunnenberg HG, Stewart AF. Heterodimerization of the Drosophila ecdysone receptor with retinoid $\mathrm{X}$ receptor and ultraspiracle. Nature 1993 ; $362: 471-5$

21. Yao TP, Forman BM, Jiang $Z$, Cherbas $L$, Chen JD, McKneown $M$, Cherbas P, Evans RM. Functional ecdysone receptor is the product of $E c R$ and Ultraspiracle genes. Nature $1993 ; 366$ : 476-9

22. Deutsch J. Ultraspiracle et le récepteur de l'ecdysone ou les relations dans la famille des récepteurs hormonaux nucléaires de la drosophile aux mammifères. médecine/sciences $1993 ; 9: 701$ 5

23. Umesono K, Murakami KK, Thompson CC, Evans RM. Direct repeats as selective response elements for the thyroid hormone, retinoic acid, and vitamin $D_{3}$ receptors. Cell 1991 ; 65 : 1255-66.

24. Durand B, Saunders M, Leroy $P$, Leid M, Chambon P. All-trans and 9-cis retinoic acid induction of CRABPII transcription is mediated by RAR-RXR heterodimers bound to DR1 and DR2 repeated motifs. Cell $1992 ; 71: 73-85$.

25. Zhang XK, Lehmann J, Hoffmann B, Dawson MI, Cameron J, Graupner G, Hermann T, Tran P, Pfahl M. Homodimer formation of retinoid $\mathrm{X}$ receptor induced by 9 -cis retinoic acid. Nature $1992 ; 358$ : 587-91.

26. Carlberg C, Bendik I, Wyss A, Meier E, Sturzenbecker LJ, Grippo JF, Hunziker W. Two nuclear signalling pathways for vitamin D. Nature $1993 ; 361$ :657-60.

27. Freemont PS, Hanson IM, Trowsdale J. A novel cysteine-rich sequence motif. Cell $1991 ; 64: 483-4$

28. Reddy BA, Etkin LE, Freemont PS A novel zinc finger coiled-coil domain in a family of nuclear proteins. Trends Biol Sci nucléiques [27]. Immédiatement en aval de ce motif, la présence d'une ou deux régions supplémentaires de type "doigt de zinc", les boîtes B, suivies d'un domaine riche en hélice $\alpha$ correspondant à une interface de dimérisation, définissent, au sein de la famille des protéines RING finger, une sous-famille pour laquelle sept membres ont été décrits jusqu'à présent [28]. Certains d'entre eux sont capables de lier les acides nucléiques, tel le facteur de transcription RPT-1 [29] ou encore la protéine A33 isolée chez le triton et qui est étroitement associée aux ARNm en cours de synthèse [30]. Deux autres membres sont, comme PML, impliqués dans des processus oncogéniques sous la forme de protéines de fusion résultant de translocations chromosomiques : les protéines transformantes T18 et RFP [31, 32]

L'utilisation d'anticorps dirigés contre la protéine PML a permis de localiser celle-ci au niveau de structures subnucléaires très particulières, dont le nombre varie entre 3 et 20 selon le type cellulaire étudié [3335]. Au niveau ultrastructural, ces foyers PML apparaissent comme des structures denses aux électrons qui adoptent souvent la forme d'un anneau et dont le diamètre oscille entre 0,3 et $0,5 \mu \mathrm{m}$. Ces structures, décrites par les microscopistes électroniciens il y a une trentaine d'années, ont reçu le nom de " corps nucléaires" et correspondent, pour certains, à de véritables organites bien qu'aucune fonction particulière ne leur ait été attribuée jusqu'à présent [5, 36]. Ils sont présents dans toutes les cellules étudiées mais leur nombre et leur taille peuvent varier en fonction d'un certain nombre de stimuli ou de stress (choc thermique, traitement à l'interféron...). Les corps nucléaires PML correspondent à des complexes multiprotéiques dont un seul des composants, mise à part la protéine PML elle-même, a été clairement caractérisé à ce jour, et dénommé protéine SP100 [37]. Cette protéine peut se comporter comme un autoantigène dans un certain nombre de maladies auto-immunes chez l'homme ; cependant, aucun rôle de nature à éclairer la fonction de PML n'a pu encore lui être assigné.

\section{Structure de la protéine de fusion PML-RAR $\alpha$}

La translocation $t(15 ; 17)$ engendre une fusion entre les gènes $P M L$ et $R A R \alpha$, localisés respectivement sur les chromosomes 15 et 17 , telle que l'expression de la protéine hybride PML-RAR $\alpha$ est placée sous le contrôle du promoteur du gène PML [3842]. Dans les cellules des LAP, on observe généralement une surabondance de la protéine PML-RAR $\alpha$ par rapport au récepteur natif. La chimère retient la majeure partie des domaines fonctionnels des deux protéines natives, notamment le RING finger, les boîtes $\mathrm{B}$ et la région en hélice $\alpha$ au sein de PML, ainsi que les domaines $\mathrm{B}$ à $\mathrm{F}$ de $\mathrm{RAR} \alpha$ (figure 2). Alors que le point de cassure sur le chromosome 17 est toujours situé au niveau du deuxième intron du gène $R A R \alpha$, ce qui a pour effet d'entraîner la délétion de la région A, deux points de cassure principaux au sein du gène $P M L$ définissent deux protéines chimériques différentes. Dans $90 \%$ des cas, la translocation s'effectue, soit au niveau du troisième intron, soit au niveau du sixième intron, engendrant respectivement les protéines PML-RAR $\alpha$ S et PML-RAR $\alpha$ L. Le fait que le produit de fusion réciproque RAR $\alpha$-PML ne soit pas présent de façon systématique chez tous les malades atteints de leucémie aiguë promyélocytaire (LAP) suggère fortement que seul l'hybride PML-RAR $\alpha$ joue un rôle majeur dans le développement de la maladie [43]. Dans quatre cas de LAP, une translocation variante de type $t(11 ; 17)$ juxtapose le gène $\mathrm{RAR} \alpha$ à un gène codant pour une protéine à doigt de zinc de la famille Krüppel, PLZF, suggérant que l'altération du récepteur est un élément crucial dans la pathogénie des LAP [44].

\section{Propriétés fonctionnelles de PML-RAR $\alpha$}

Les mécanismes par lesquels l'hybride PML-RAR $\alpha$ exerce son effet dominant négatif sur la différenciation promyélocytaire sont encore mal connus. Il est probable que PML-RAR $\alpha$ interfère directement avec les fonctions des protéines RAR $\alpha$ et PML. Parallèlement, 


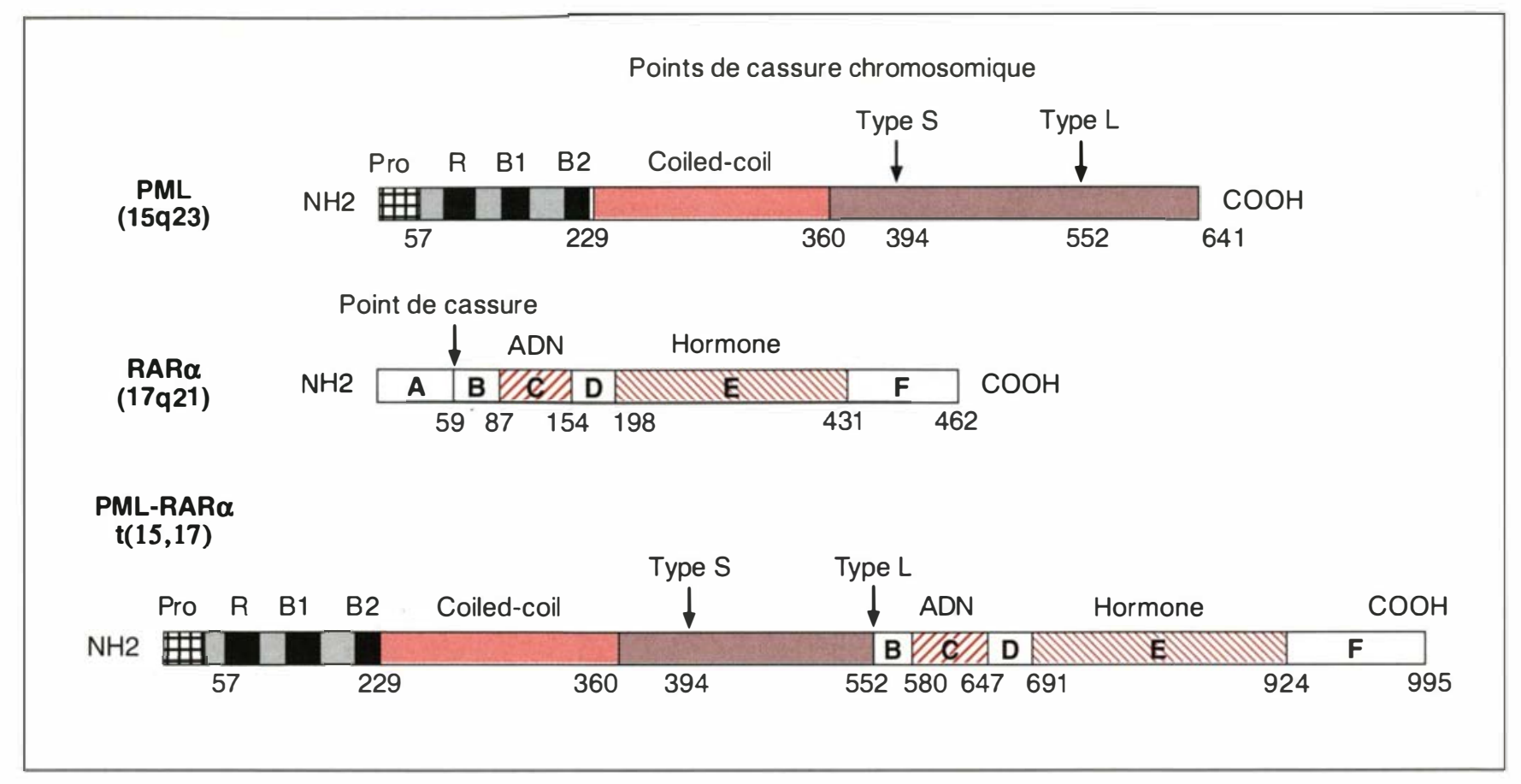

Figure 2. Structure des protéines PML et RAR $\alpha$ natives et des deux types de protéines de fusion PML-RAR $\alpha$ engendrées par la translocation $\mathbf{t}(15 ; 17)$. Les principaux domaines fonctionnels candidats de $P M L$ et $R A R \alpha$ sont représentés. Au sein de la protéine PML, on distingue un domaine amino-terminal riche en résidus proline (Pro), le motif de type RING finger $(R)$, les deux boîtes riches en résidus cystéine $\left(B_{1}\right.$ et $\left.B_{2}\right)$ et la région à hélice $\alpha$ correspondant à un domaine de dimérisation. Au sein de $R A R \alpha$, on distingue la région $A V B$, modulateur de la transcription, la région $C$ qui correspond au domaine de liaison à l'ADN, la région $E$ qui contient le domaine de fixation de l'hormone et renferme aussi le domaine transactivateur et le domaine de dimérisation, les régions $D$ et $F$ qui n'ont pas encore de fonction bien définie. Les chiffres représentent la position des acides aminés définissant les limites de ces domaines. La protéine hybride PML-RAR $\alpha$ retient la majeure partie des domaines fonctionnels des deux protéines natives. Deux points de cassure principaux, $S$ et $L$, au sein du gène PML définissent deux protéines chimères différentes. Le point de cassure sur le chromosome 17 est toujours situé au niveau du deuxième intron du gène RAR $\alpha$, ce qui a pour effet d'entraîner la délétion de la région $A$.

l'hybride peut avoir acquis des propriétés qui lui sont propres, inhibant alors la fonction d'autres protéines impliquées dans la différenciation myéloïde.

Sur les gènes cibles de l'AR, PMLRAR $\alpha$ présente des propriétés trans activatrices altérées comparées à celles du récepteur natif ; cependant, le phénotype de l'hybride est variable selon les types de cellule et de gène cible étudiés. Ainsi, en présence d'AR, PML-RAR $\alpha$ peut se comporter, soit comme un fort répresseur, soit comme un superactivateur de la transcription [38, 39]. La signification biologique d'un comportement aussi versatile demeure obscure. L'hybride PML-RAR $\alpha$ possède, par ailleurs, un phénotype radicalement opposé à celui du récepteur $\operatorname{RAR} \alpha$ en ce qui concerne son aptitu- de à interférer avec la voie transcriptionnelle relayée par les oncogènes jun et fos (activité "APl »). Ainsi, contrairement à $\mathrm{RAR} \alpha$ qui, en présence d'acide rétinoïque, inhibe l'expression des gènes sensibles au complexe AP1, l'hybride PML-RAR $\alpha$ les active fortement [45]. Sachant qu'une élévation de l'activité APl accompagne généralement la différenciation hématopoïétique, il est possible que l'interférence de PMLRAR $\alpha$ avec cette voie d'activation puisse contribuer à l'effet thérapeutique de l'AR dans ce type de leucémie.

L'interface de dimérisation, constituée par les hélices $\alpha$ de la partie PML au sein de l'hybride PML$\operatorname{RAR} \alpha$, est capable de conférer à celui-ci la propriété de former des homodimères stables en solution. En l'absence de RXR, de tels homodimères PML-RAR $\alpha$ peuvent lier l'ADN ; cependant, la spécificité de liaison de ces complexes vis-à-vis de certains éléments de réponse hormonaux, notamment les répétitions directes séparées de 1 nucléotide, est différente de celle des hétérodimères RAR/RXR ou PML-RAR/RXR [46]. Ainsi, par le jeu de ces homodimères, dont on a pu prouver l'existence réelle dans des extraits nucléaires de cellules LAP (J. Jansen et A. Dejean, manuscrit soumis), l'hybride PML-RAR $\alpha$ pourrait interférer avec des voies hormonales multiples.

Si la protéine PML se concentre au niveau de corps nucléaires bien définis au sein du noyau, l'hybride PMLRAR $\alpha$, présent dans les cellules LAP, est délocalisé au niveau de structures 
29. Patarca R, Schwartz J, Singh RP Kong KT, Murphy E, Anderson Y, Sheng FY, Singh P, Johnson KA, Guarnagia SM, Durfee T, Blattner F, Cantor H. rpt-1 : an intracellular protein from helper inducer $T$ cells that regulates gene expression from interleukin 2 receptor and human immunodeficiency virus type 1. Proc Natl Acad Sci USA $1988 ; 85: 2733-7$.

30. Bellini M, Lacroix JC, Gall JG. A putative zinc-binding protein on lampbrush chromosome loops. EMBO J 1993 ; 12 : 107 14.

31. Miki T, Fleming TP, Crescenzi M, Molloy CJ, Blam SB, Reynolds SH, Aaronson SA. Development of a highly efficient expression cDNA cloning system : application to oncogene isolation. Proc Natl Acad Sci USA 1991 ; 88 : 5167-71.

32. Takahashi $\mathrm{M}$, Inaguma $\mathrm{Y}$, Hiai $\mathrm{H}$, Hirose F. Developmentally regulated expression of a human "finger "containing gene encoded by the 5 half of the ret transforming gene. Mol Cell Biol $1988 ; 8: 1853-6$.

33. Weis K, Rambaud S, Lavau C, Jansen J, Carvalho T, Carmo-Fonseca M, Lamond A, Dejean A. Retinoic acid regulates aberrant nuclear localization of PML-RAR $\alpha$ in acute promvelocytic leukemia cells. Cell 1994; 76 : 345-56.

34. Dyck JA, Maul GG, Miller WH, Chen JD, Kakizuka A, Evans RM. A novel macromolecular structure is a target of the promyelocyte retinoic acid receptor oncoprotein. Cell $1994 ; 76: 333-43$.

35. Koken MHM, Puvion-Dutilleul F, Guillemin MC, Viron A, Linares-Cruz G, Stuurman N, De Jong L, Szostecki C, Calvo F, Chomienne C, Degos L, Puvion E, de The $H$. The $t(15 ; 17)$ translocation alters a nuclear body in a retinoic acid-reversible fashion. $E M B O J 1994 ; 13$ : 1073-83.

36. Bouteille M, Laval M, Dupuy-Coin AM. Localization of nuclear functions. In : Busch H, ed. The Cell Nucleus. New York Academic Press, $1974 ; 1$ : 5-64.

37. Szostecki C, Guldner HH, Netter HJ, Will $\mathrm{H}$. Isolation and characterization of cDNA encoding a human nuclear antigen predominantly recognized by autoantibodies from patients with primary biliary cirrhosis. J Immunol $1990 ; 145: 4338$-47.

38. De Thé H, Lavau C, Marchio A, Chomienne C, Degos L, Dejean A. The PML-RAR $\alpha$ fusion mRNA generated by the $t(15 ; 17)$ translocation in acute promyelocytic leukemia encodes a functionally altered RAR. Cell 1991; 66: $675-84$.

39. Kakizuka A, Miller WH, Umesono K, Warrell RP, Frankel SR, Murty VVVS, Dmitrovsky E, Evans RM. Chromosomal translocation $\mathrm{t}(15 ; 17)$ in human acute promyelocytic leukemia fuses RAR $\alpha$ with a novel putative transcription factor, PML.
40. Kastner P, Perez A, Lutz Y, RochetteEgly C, Gaub MP, Durand B, Lanotte M, Berger $R$, Chambon P. Structure, localization and transcriptional properties of two classes of retinoic acid receptor a fusion proteins in acute promyelocytic leukemia (APL) : structural similarities with a new family of oncoproteins. $E M B O J$ $1992 ; 11: 629-42$.

41. Pandolfi PP, Grignani F, Alcalay M Mencarelli A, Biondi A, LoCoco $\mathrm{F}$ Grignini F, Pelicci PG. Structure and origin of the acute promyelocytic leukemia myl/RAR $\alpha$ cDNA and characterization of its retinoid-binding and transactivation properties. Oncogene $1991 ; 6: 1285-92$.

42. Goddard AD, Borrow J, Freemont PS Solomon E. Characterization of a zinc finger gene disrupted by the $t(15 ; 17)$ in acute promyelocytic leukemia. Science 1991 ; $254: 1371-4$

43. Alcalay M, Zangrilli D, Fagioli M Pandolfi PP, Mencarelly A, Lo Coco F, Biondi A, Grignani F, Pelicci P. Expression pattern of the RAR $\alpha$-PML fusion gene in acute promyelocytic leukemia. Proc Nat Acad Sci USA $1992 ; 89$ : 4840-4.

44. Chen Z, Brand NJ, Chen A, Chen SJ Tong JH, Wang $Z Y$, Waxman S, Zelent $A$ Fusion between a novel Krüppel-like zinc finger gene and the retinoic acid receptor $\alpha$ locus due to a variant $\mathrm{t}(11 ; 17)$ translocation associated with acute promyelocytic leukaemia. EMBO J $1993 ; 12$ : 1161-7.

45. Doucas V, Brockes IP, Yaniv M, De The $H$, Dejean $A$. The PML-retinoic acid receptor $\alpha$ translocation converts the receptor from an inhibitor to a retinoic acid-dependent activator of transcription factor AP1. Proc Natl Acad Sci USA 1993; 90 9345-9.

46. Perez A, Kastner P, Sethi S, Lutz Y Reibel C, Chambon P. PML-RAR homodimers, distinct DNA binding properties and heteromeric interactions with RXR. EMBO J 1993 ; 12 : 3171-82.

47. Testa $\mathbf{U}$, Masciulli R, Tritarelli E, Pustorino R, Mariani G, Martucci R, Barberi $T$, Camagna A, Valtieri M, Peschle C. Transforming growth factor- $\beta$ potentiates vitamin $D$ induced terminal monocytic differentiation of human leukemic cell lines. J Immunol 1993; $150: 2418$.

48. Grignani F, Ferrucci PF, Testa U Talamo G, Fagioli M. Alcalay M, Mencarell A, Grignani F, Peschle C, Nicoletti I, Pelicci PG. The acute promyelocytic leukemiaspecific PML-RAR $\alpha$ fusion protein inhibits differentiation and promotes survival of myeloid precursor cells. Cell $1993 ; 74: 423$ 31 .

49. Rousselot P, Hardas B, Patel A Guidez F, Gäken J, Castaigne S, Dejean A De Thé H, Degos L, Farzaneh F, Chomienne C. The PML-RAR $\alpha$ gene product of the $t(15 ; 17)$ translocation inhibits retinoic acid-induced granulocytic differentiation and mediated transactivation in human myeloid cells. Oncogene $1994 ; 9: 545-51$. anormales, désorganisées, étroitement associées à la chromatine, donnant un profil d'immunofluorescence microponctué caractéristique [3335]. La chimère est, par ailleurs, capable d'entraîner dans sa délocalisation la protéine RXR (pour revue, voir [5]) (figure 3), ainsi qu'un certain nombre de protéines normalement associées aux corps nucléaires, telles que les protéines PML et SPI00 [3335]. Ainsi, l'effet dominant négatif de PML-RAR $\alpha$ pourrait s'exercer par le biais d'une séquestration de ces protéines loin de leur site d'action naturel. Si les effets d'une telle délocalisation sur la fonction naturelle des corps nucléaires sont encore inconnus, il est, en revanche, aisé d'imaginer que la titration du partenaire RXR puisse affecter, non seulement la fonction des RAR, mais aussi celle de tous les récepteurs nucléaires dont l'activité dépend de leur interaction avec RXR au sein d'hétérodimères. Parmi ceux-ci, un autre candidat susceptible d'être "inactivé " indirectement par la chimère est le récepteur de la vitamine $D_{3}$. En effet, comme l'AR, la vitamine $\mathrm{D}_{3}$ est capable d'induire la différenciation des cellules myéloïdes. Des travaux récents ont permis de démontrer que la protéine PML-RAR $\alpha$ est effectivement capable d'inhiber à la fois la liaison du récepteur de la vitamine $D_{3}$ (VDR) à l'ADN in vitro et l'activation d'un gène indicateur contrôlé par des éléments d'ADN conférant la réponse à la vitamine $\mathrm{D}_{3}$ dans des cellules transfectées [46]. Dans ces deux cas, il a été possible de montrer que le blocage de l'activité du VDR est bien dû à une compétition pour le produit RXR.

\section{PML-RAR $\alpha$ et ses effets sur la différenciation}

Jusqu'à présent, les tentatives d'exprimer PML-RAR $\alpha$ dans les cellules précurseurs de la moelle afin d'évaluer ses propriétés oncogéniques ont échoué. La toxicité de l'hybride observée dans un grand nombre de types cellulaires pourrait être, en partie, responsable de ces échecs. Cependant, certaines lignées cellulaires en culture peuvent tolérer l'expression stable de la chimère, par exemple la lignée U937. Les cellules promonocytiques U937 ont la capa- 

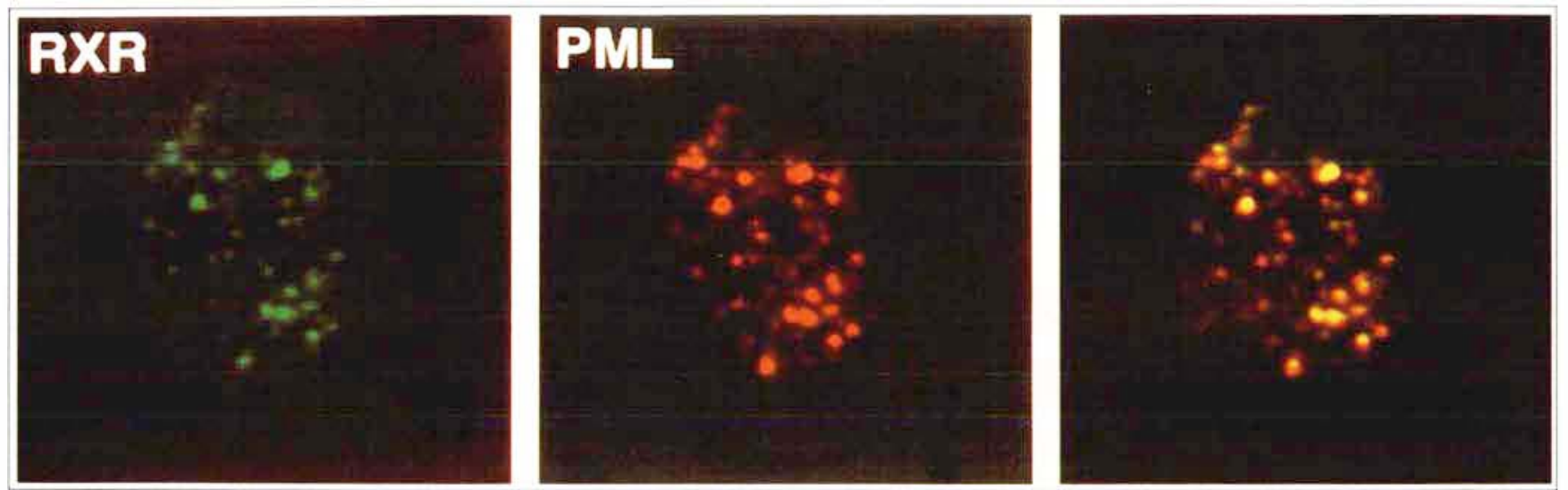

Figure 3. Colocalisation de PML-RAR $\alpha$ et RXR au niveau des structures anormales dans un noyau d'une cellule de leucémie aiguë promyélocytaire (LAP). Des cellules de LAP sont soumises à un double marquage en immunofluorescence puis photographiées à travers un microscope confocal. Les marquages obtenus avec les anticorps dirigés contre les protéines $R X R$ et $P M L-R A R \alpha$ apparaissent de couleur verte et rouge respectivement. La correspondance entre les deux marquages est représentée en jaune.

cité de se différencier en monocytes sous l'effet de la vitamine $\mathrm{D}_{\text {, }}$, seule ou associée au facteur TGFß1 [47]. De plus, un faible pourcentage de cellules U937 peut se différencier sous l'effet de faibles doses $\left(10^{-9} \mathrm{M}\right)$ d'AR. Lorsque l'hybride PMI-RARo est exprimé de façon stable dans les cellules U937, on observe un blocage de leur différenciation : les cellules sont devenues résistantes aux traitements par la vitamine $\mathrm{D}_{3}$ et par l'AR. En revanche, lorsque ces mêmes cellules exprimant l'hybride sont exposées à de fortes doses d'AR $\left(10^{-6} \mathbf{M}\right)$, proches des doses thérapeutiques, le pourcentage de cellules qui entrent dans le programme de différenciation devient supérieur à celui des cellules U937 témoins [48]. Tout se passe comme si, en présence de fortes concentrations d'AR, l'hybride, jusqu'alors inhibiteur, devenait un élément stimulateur de la différenciation. Des résultats comparables ont été observés dans la lignée myéloïde HL60. L'expression transitoire de la chimère PML-RAR est en effet capable de bloquer la différenciation granulocytaire normalement induite par l'AR, alors que de fortes doses d'AR lèvent ce blocage, corroborant l'effet thérapeutique de l'AR dans ce type d'hémopathie [49].

\section{Conclusion}

Quels sont les mécanismes molécu$\mathrm{m} / \mathrm{s} n^{\circ} 8 / 9$ vol. 10 , août-septembre 94 laires à l'origine du blocage des promyélocytes à un stade précoce de leur différenciation au cours des leucémies aiguës promvélocytaires et comment ce phénotype peut-il s'inverser lors du traitement par l'acide rétinoïque? L'identification d'un récepteur de l'acide rétinoïque altéré sous forme d'une protéine de fusion PML-RAR $\alpha$ dans tous les cas de IAP présentant la translocation $\mathrm{t}(15 ; 17)$ apporte la première démonstration de l'implication d'un récepteur nucléaire dans un cancer chez l'homme et, par là même, un début de réponse à ces questions. Plusieurs types de travaux émanant de laboratoires différents ont maintenant permis de démontrer clairement que l'hybride peut interférer avec un certain nombre de voies d'activation transcriptionnelle, telles que les voies RAR, mais aussi RXR, TR, VDR et, plus indirectement, API. La formation d'homodimères PML-RAR $\alpha$ ayant une spécificité de reconnaissance altérée ainsi que la titration du partenaire RXR par l'hybride ont été évoquées.

Par ailleurs, il est aussi très probable que, par sa localisation intranucléaire aberrante et par sa capacité de s'associer fortement avec PML, PMLRAR $\alpha$ puisse interférer parallèlement avec les fonctions normales de cette protéine et avec celles des corps nucléaires en général. La déstabilisation, le "détournement " d'un certain nombre de protéines entrant dans la composition de ces structures subnucléaires pourrait être en cause.

Sans fournir d'explications réelles, l'observation d'une réorganisation complète des structures intranucléaires PML-RAR $\alpha$ après traitement des cellules de LAP par l'AR offre un parallèle frappant à l'effet thérapeutique de ce rétinoïde. En effet, en présence d'AR, un certain nombre de protéines telles que RXR, PML et SPIO0) retrouvent leur localisation intranucléaire habituelle, caractéristique des cellules non leucémiques promyélocytaires. Ainsi le rôle physiologique des composants des corps nucléaires serait intimement lié à leur localisation précise au sein du noyau. Dans l'hypothèse où ces structures joueraient effectivement un rôle dans la différenciation myéloïde, alors l'hybride PMI-RAR $\alpha$ fournirait une parfaite illustration de l'interdépendance entre des mécanismes multiples impliqués dans une même voie de différenciation

\section{TIRÉS A PART}

A. Dejean.

Remerciements

Les auteurs tiennent à remercier le Prof esseur P. Tiollais pour son soutien constant. 


\section{Summary}

Paradoxical effect of retinoic acid in acute promyelocytic leukaemia

Retinoic acid (RA) is a vitamin A deriva tive with striking effects on development and cell differentiation. The identifica tion of three $\mathrm{RA}$ receptors $(\mathrm{RAR} \alpha, \beta$ and $\gamma$ ) as members of the nuclear receptor superfarnily led to important insights into the molecular mechanism of action of retinoids. The nuclear receptors, including also receptors for steroid hormone, vitamine $\mathrm{D}_{3}$ and thyroid homone, act as ligand-inducible transcription factors and are characterized by the presence of two well conserved DNA - and hormone-binding domains. One of the most intriguing properties of RA is its ability to induce in vivo differentiation of acute promyelocytic leukaemia (APL) cells into mature granulocytes, leading to complete morphological remissions. We and others have shown that the $\mathrm{t}(15 ; 17)$ translocation, specifically associated with APL, fuses an as yet unidenti- fied gene, named $P M L$, to the retinoic acid receptor $\alpha$ locus. The resulting PML-RAR $\alpha$ hybrid protein that retains most of the functional domains of parental proteins exhibits altered transactivating functions when compared to the wild-ype receptor; however, the biological significance of this property in the transforming phenotype is still obs cure. PML, whose function is unknown, belongs to a novel farnily of nuclear proteins characterized by the presence of a Cys/Hisrich motif, named a RING finger, that includes RNA-binding proteins, transcription factors and oncoproteins. A dimenzation domain within PML is able to mediate the formation of PML RAR $\alpha$ homodimers that can bind to target sequences with distinct DNA binding properties if compared with $R A R \alpha$. Immunofluorescence studies have shown that PML is specifically localized within a discrete subnuclear compartment corresponding to nuclear bodies recognized by patient autoimmune sera. These structures are distinct from snRNP-containing organelles. In APL cells, the PMLRAR $\alpha$ hybrid that accumulates into abnormal substructures is able to delocalize the natural $\mathrm{RAR} \alpha$ partner, RXR, as well as some of the protein components of the nuclear bodies. This suggests that PML-RAR $\alpha$ could act as a dominant negative oncoprotein by diverting a subset of proteins from their natural sites of action. Interestingly, RA treatment induces a complete relocalization of each of these proteins leading to the proposal that the paradoxical therapeutic effect of RA in promoting myeloid differentiation in APL might be related to its ability to restore a normal subnuclear organization. 